\title{
Utilidad diagnóstica de la tomografía de coherencia óptica en pacientes con neuritis óptica desmielinizante
}

\section{Diagnostic utility of optical coherence tomography (OCT) in patients with demyelinating optic neuritis}

\author{
Carolina Ossa-Calderón ${ }^{1}$, Eduardo J. Polanía-Barón ${ }^{1 *}$, Héctor D. Forero ${ }^{1}$, Patricia Quintero-Cusguen², \\ Diego A. Jaimes ${ }^{3}$ y Natalia Valenzuela ${ }^{3}$
}

${ }^{1}$ Servicio de Oftalmología, Hospital Universitario de La Samaritana E.S.E., Bogotá D.C.; ${ }^{2}$ Servicio de Neurología, Hospital Universitario de La Samaritana E.S.E., Bogotá D.C.; ${ }^{3}$ Facultad de Medicina, Universidad de La Sabana, Chía. Colombia

\section{Resumen}

Objetivo: Determinar las diferencias en el grosor de la capa de las fibras nerviosas (CFN) por medio de la tomografía de coherencia óptica (OCT) en pacientes con neuromielitis óptica (NMO) y esclerosis múltiple (EM). Métodos: Estudio de serie de casos en pacientes adultos con diagnóstico de EM y NMO. Resultados: La mediana estimada y el rango intercuartílico (RIQ) para el grosor de la CFN en personas sanas fue de $110 \mathrm{RIQ}$ (16) $\mu \mathrm{m}$, en los pacientes con EM fue de 94 (21) $\mu m$ y en los pacientes con NMO fue de 76.5 (61) $\mu \mathrm{m}$. Se estimaron las diferencias entre pacientes sanos y enfermos, y se encontró que el grosor de la CFN era significativamente inferior en datos absolutos: $\Delta=16 \mu \mathrm{m}$ para sanos-EM y $\Delta=33.5$ um para sanos-NMO. Respecto a los cuadrantes de CFN, se encontró que los cuadrantes inferiores y superiores tienen mayor pérdida de fibras nerviosas en ambas enfermedades. Conclusiones: Nuestro estudio soporta evidencia relacionada con el compromiso de la capa de células ganglionares y CFN observado en pacientes con EM y NMO. Este estudio nos confirma lo reportado en otros estudios, que el grosor de la CFN es una medida que puede ayudar a diagnosticar el compromiso y la gravedad del nervio óptico que puede estar afectado en estas dos enfermedades desmielinizantes, con patrones en los que se puede diferenciar entre NMO y EM.

Palabras claves: Neuromielitis óptica. Esclerosis múltiple. Neuritis óptica.Tomografía. Coherencia óptica. Fibras nerviosas.

\begin{abstract}
Objective: To determine differences in nerve fiber layer (NFL) thickness by Optical Coherence Tomography (OCT) in patients with neuromyelitis optica (NMO) and Multiple Sclerosis (MS). Methods: Case series study in adult patients diagnosed with MS and NMO. Results: The estimated median and interquartile range (IQR) for nerve fiber layer thickness in healthy subjects was 110 IQR (16) $\mu \mathrm{m}$, in patients with MS was 94 (21) $\mu \mathrm{m}$ and in patients with NMO was 76.5 (61) $\mu \mathrm{m}$. The differences between healthy and sick subjects were estimated, finding that the CFN thickness was significantly lower in absolute data: $\Delta=16 \mu \mathrm{m}$ for healthy-MS and $\Delta=33.5 \mu \mathrm{m}$ for healthy-NMO. Regarding the quadrants of retinal nerve fiber layer, it was found that the lower and upper quadrants have a greater loss of nerve fibers in both diseases. Conclusions: Our study supports evidence related to the involvement of ganglion cell layer and NFL observed in patients with MS and NMO. This study con-
\end{abstract}

Correspondencia:

*Eduardo José Polanía-Barón

C. Maestro Antonio Caso, 60, apto $201 \quad$ Fecha de recepción: 23-09-2018

Ciudad de México, México Fecha de aceptación: 07-12-2018

Disponible en internet: 01-03-2019

Fecha de aceptación: 07-12-2018 Rev Mex Oftalmol. 2019;93(2):69-74

E-mail: epoba16@hotmail.com

DOI: 10.24875/RMO.M18000062 www.rmo.com.mx

0187-4519/C 2018 Sociedad Mexicana de Oftalmología. Publicado por Permanyer México. Este es un artículo Open Access bajo la licencia CC BY-NC-ND (http://creativecommons.org/licenses/by-nc-nd/4.0/). 
Rev Mex Oftalmol. 2019;93

firms what has been reported in other studies, that NFL thickness is a measure that can help diagnose the compromise and severity of optic nerve involvement in these two demyelinating diseases, with patterns that help to differentiate between NMO and $E M$.

Key words: Neuromyelitis optica. Multiple sclerosis. Optic neuritis. Tomography. Optical coherence. Nerve fibers.

\section{Introducción}

La esclerosis múltiple (EM) y la neuromielitis óptica (NMO) son un espectro de enfermedades inflamatorias, autoinmunes, desmielinizantes del sistema nervioso central ${ }^{1,2}$. Estas dos patologías tienen una baja prevalencia en nuestra población, pero una alta incidencia de alteraciones oftalmológicas, entre las cuales se encuentran la pérdida de la visión monocular y la degeneración neuronal a nivel de la retina y el nervio óptico ${ }^{3,4}$.

La utilización de la tomografía de coherencia óptica (OCT) en pacientes con enfermedades desmielinizantes como la EM y la NMO es de gran importancia en la evaluación diagnóstica y de progresión de estas enfermedades, además, permite establecer el grosor de las capas de fibras nerviosas (CFN), el cual determinará el pronostico visual del paciente, su calidad de vida y la progresión de su enfermedad causal ${ }^{4}$. La OCT es un método innovador de alta resolución no invasivo que mide el grosor de la CFN de la retina ${ }^{5}$, da información acerca de la topografía de la cabeza del nervio óptico, el grosor de las CFN peripapilares y el volumen macular.

La OCT que se utiliza actualmente es la de tipo Time-Domain, que provee un corte seccional en tiempo real de la imagen de las capas de la retina con una resolución de $10 \mu \mathrm{m}$ (OCT Stratus $₫$ Zeiss). La desventaja de este equipo es la baja resolución y la velocidad lenta (400 A-scans/sec), lo que resulta en una medición menos precisa de las capas de la retina, comparado con otros dispositivos de $\mathrm{OCT}^{6}$. El RS$3000 ®$ (NIDEK@) es la OCT más reciente que utiliza la detección tipo Spectral-Domain (SD), el cual se encuentra disponible y comercialmente aprobado por la Food and Drug Administration. También adquiere la imagen en tiempo real, con una resolución de $4 \mu \mathrm{m}$ y una velocidad de escaneo de $53.000 \mathrm{~A}$-scans/sec, mejorando la resolución e identificación de las capas de la retina?.

El objetivo de nuestro proyecto es caracterizar el grosor de la CFN en pacientes con antecedentes de EM y NMO, por medio del nuevo dispositivo OCT RS-3000® (NIDEK@) y establecer las diferencias en dicho grosor al comparar los resultados con pacientes sanos.

\section{Métodos}

Este estudio se realizó dentro de las normas éticas consideradas en la declaración de Helsinki. Se realizó y aplicó el consentimiento informado con aprobaciones de del Comité de Ética en Investigación del Hospital Universitario de la Samaritana (05IC10-V1), Bogotá, Colombia.

Se reclutaron sujetos mayores de 18 años de manera consecutiva en tres grupos: Pacientes que tuvieran diagnóstico por historia clínica de EM o NMO, independientemente del tiempo de evolución; pacientes sin neuritis óptica activa con un tiempo mayor a un año desde el último evento de neuritis óptica, y sujetos sanos. Para los pacientes con EM y NMO se realizó una búsqueda a partir del archivo de historias clínicas del Servicio de Neurología y de Oftalmología del Hospital Universitario de La Samaritana, recolectando 35 posibles candidatos para el estudio. A partir de las historias clínicas se verificó el cumplimiento de los criterios clasificatorios de McDonald 2010 y bandas oligoclonales para $\mathrm{EM}^{8,9}$; y los criterios de La Clínica Mayo actualizados en el 2006 para $\mathrm{NMO}^{10}$; a partir de lo cual se obtuvieron 8 pacientes con EM y 2 con NMO. Los pacientes con diagnóstico de NMO estaban en controles por neurología, en tratamiento de mantenimiento con prednisolona asociada a azatioprina, un inmunosupresor. Sin embargo, la mayoría de los pacientes se encontraban en tratamiento con rituximab, un anticuerpo monoclonal que se utiliza en infusión intravenosa cada 6 meses. Los pacientes con diagnóstico de EM se encontraban con medicamentos de primera línea como interferón $\mathrm{B} 1 \mathrm{a}$, interferón $\mathrm{B} 1 \mathrm{~b}$ y medicamentos de segunda línea como fingolimod y natalizumab.

Con el fin de realizar las comparaciones entre grupos, se seleccionaron pacientes que por interrogatorio no tuvieran antecedentes de NMO ni EM. Se excluyeron pacientes que no aceptaron participar en el estudio y con comorbilidades que pudieran afectar el grosor de la CFN (diabetes mellitus, oclusiones vasculares, retinitis por $\mathrm{VIH}$, coriorretinitis, glaucoma), que tuvieran neuritis óptica activa, recaída de síntomas de la enfermedad o presencia de lesiones nuevas en imagen de resonancia magnética nuclear y pacientes con datos 
Tabla 1. Descripción de las principales características demográficas

\begin{tabular}{|l|c|c|c|}
\hline Ítem & Pacientes sanos & Esclerosis múltiple & Neuromielitis óptica \\
\hline Número de ojos & 20 & 16 & 4 \\
\hline Edad mediana (RIQ/mín/máx.) & $35.5(15 / 18 / 52)$ & $35(11 / 24 / 51)$ & $35(34 / 18 / 52)$ \\
\hline Mujeres (n) & 12 & 8 & 4 \\
\hline Tiempo de diagnóstico (años) & N/A & $5(9 / 1 / 17)$ & $2(2 / 1 / 3)$ \\
\hline Número de recaídas (años) & N/A & $1(1.5 / 0 / 2)$ & $1(0 / 1 / 1)$ \\
\hline
\end{tabular}

RIQ: rango intercuartílico.

incompletos en la historia clínica o en los registros de la base de datos. La recolección de la información consistió en la selección de pacientes que hayan asistido al Hospital Universitario de la Samaritana del 1 de enero del año 2012 al 31 de julio de 2016. Una vez establecido el cumplimiento de los criterios clasificatorios, se estableció comunicación con los pacientes para explicarles el protocolo y la firma del consentimiento informado y verificar la disponibilidad para la realización del examen.

A los pacientes disponibles se les ofreció una consulta oftalmológica completa en la que estaba incluida la OCT, que toma de manera automática el grosor de las capas de la retina. Se realizó el análisis estadístico descriptivo y se reportaron frecuencias y proporciones para variables cualitativas y para variables continuas medianas y rangos intercuartiles. Se evaluaron las diferencias por medio de la prueba de hipótesis entre las medidas correspondientes a la CFN y el grosor de la capa de células ganglionares entre los pacientes con la enfermedad y sin la enfermedad. Al cumplir criterio de no normalidad, se realizó contraste de medias con la prueba de rangos de Wilcoxon, considerando el nivel de significancia menor a 0.005 . El análisis mencionado se realizó por medio del software STATA versión 12.

\section{Resultados}

El estudio incluyó 10 pacientes para un total de 20 ojos, 16 ojos con EM y 4 ojos con NMO (Tabla 1). No se encontraron diferencias entre los tres grupos en cuanto a edad, género, tiempo de diagnóstico y número de episodios de neuritis óptica previos.

Se evaluaron las diferencias absolutas entre las medianas correspondientes a la CFN y la capa de células ganglionares entre los pacientes con la enfermedad y sin la enfermedad. La mediana (rango intercuartílico) estimada para el grosor de la CFN en personas sanas fue de 110 (16) $\mu \mathrm{m}$, para pacientes con NMO, fue de 76.5 (61) $\mu \mathrm{m}$, y para pacientes con EM, fue de 94 (21) $\mu \mathrm{m}$. Los pacientes con NMO tuvieron valores significativamente inferiores en datos absolutos en comparación con las personas con EM (Fig. 1).

Examinando los cuadrantes de las CFN (Fig. 2), se encontró que los cuadrantes inferiores y superiores tienen mayor pérdida de fibras nerviosas en ambas enfermedades. Respecto a los cuadrantes de capa de células ganglionares, se evidenció que hay mayor pérdida de células ganglionares en todos los cuadrantes en los pacientes con NMO en comparación con los pacientes con EM. Además, existe una mayor pérdida de estas en el cuadrante inferonasal en los pacientes con EM y superonasal en los pacientes con NMO (Fig. 3).

\section{Discusión}

Los estudios histopatológicos acerca de la NMO han revelado que esta enfermedad tiene un componente tanto inflamatorio como isquémico que conlleva una mayor respuesta inflamatoria y destrucción axonal ${ }^{11}$. Charcot, et al. demostraron que en la EM hay una desmielinización con una preservación axonal relativa ${ }^{12}$. Basándose en esto y en nuestros hallazgos, se infiere que al medir el grosor de la CFN en un paciente con sospecha de enfermedad desmielinizante, este seria mucho menor en pacientes con NMO que con EM, como se ha reportado previamente ${ }^{13-15}$.

Nuestro estudio sugiere la pérdida del grosor de la CFN y de la capa de células ganglionares en pacientes con enfermedad desmielinizante, específicamente en pacientes con EM y NMO. Además, se encontró que el cuadrante superior e inferior de las CFN fueron afectados en mayor magnitud en la NMO que en la EM ${ }^{13,16}$. Sin embargo, es importante aumentar el número de pacientes a largo plazo para aumentar el poder de estos resultados. 


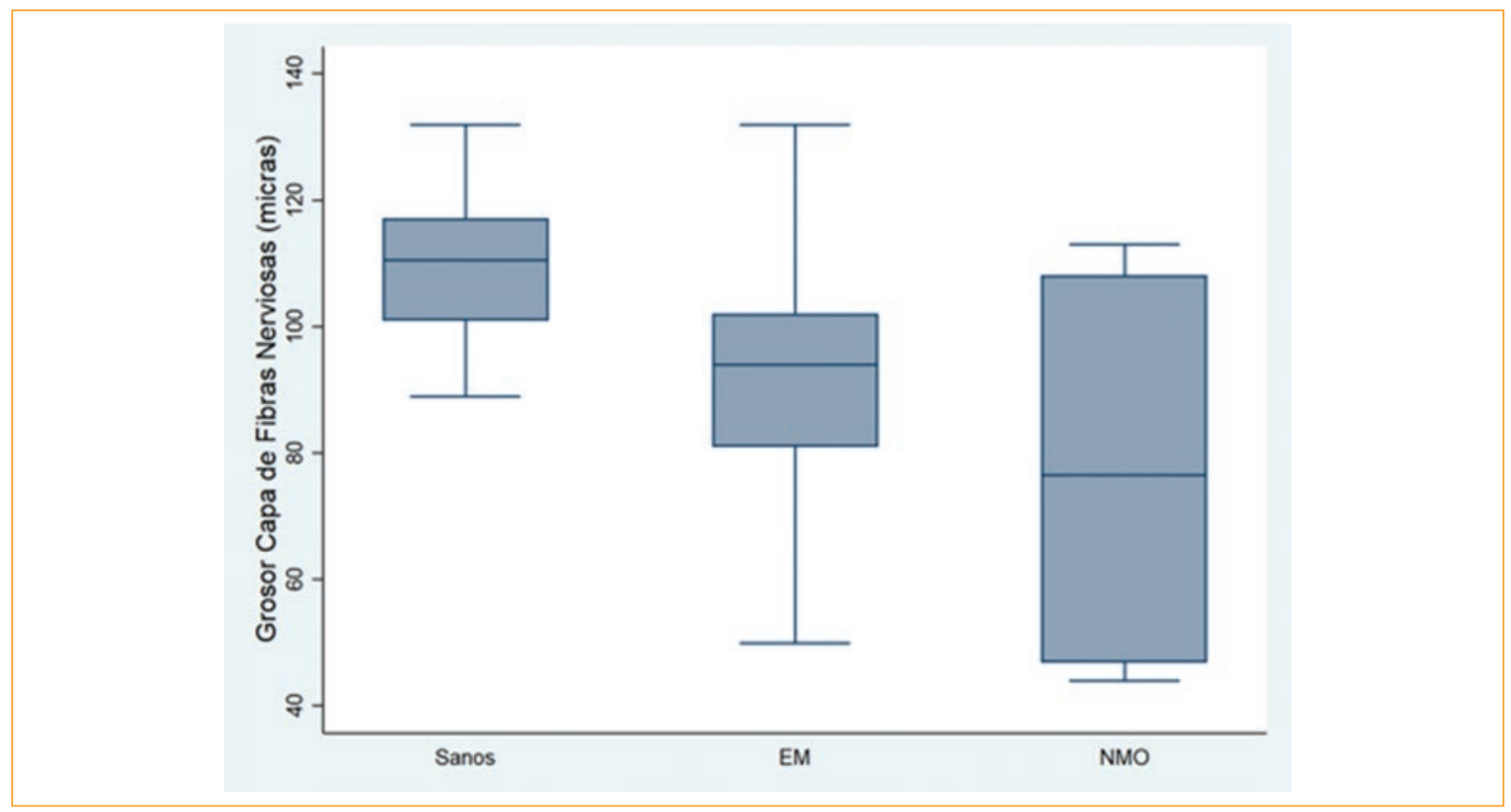

Figura 1. Grosor de la capa de fibras nerviosas en $\mu \mathrm{m}$ de acuerdo al diagnóstico $(0=\operatorname{sanos}, 1=\mathrm{EM}, 2=\mathrm{NM})$. EM: esclerosis múltiple; NMO: neuromielitis óptica.

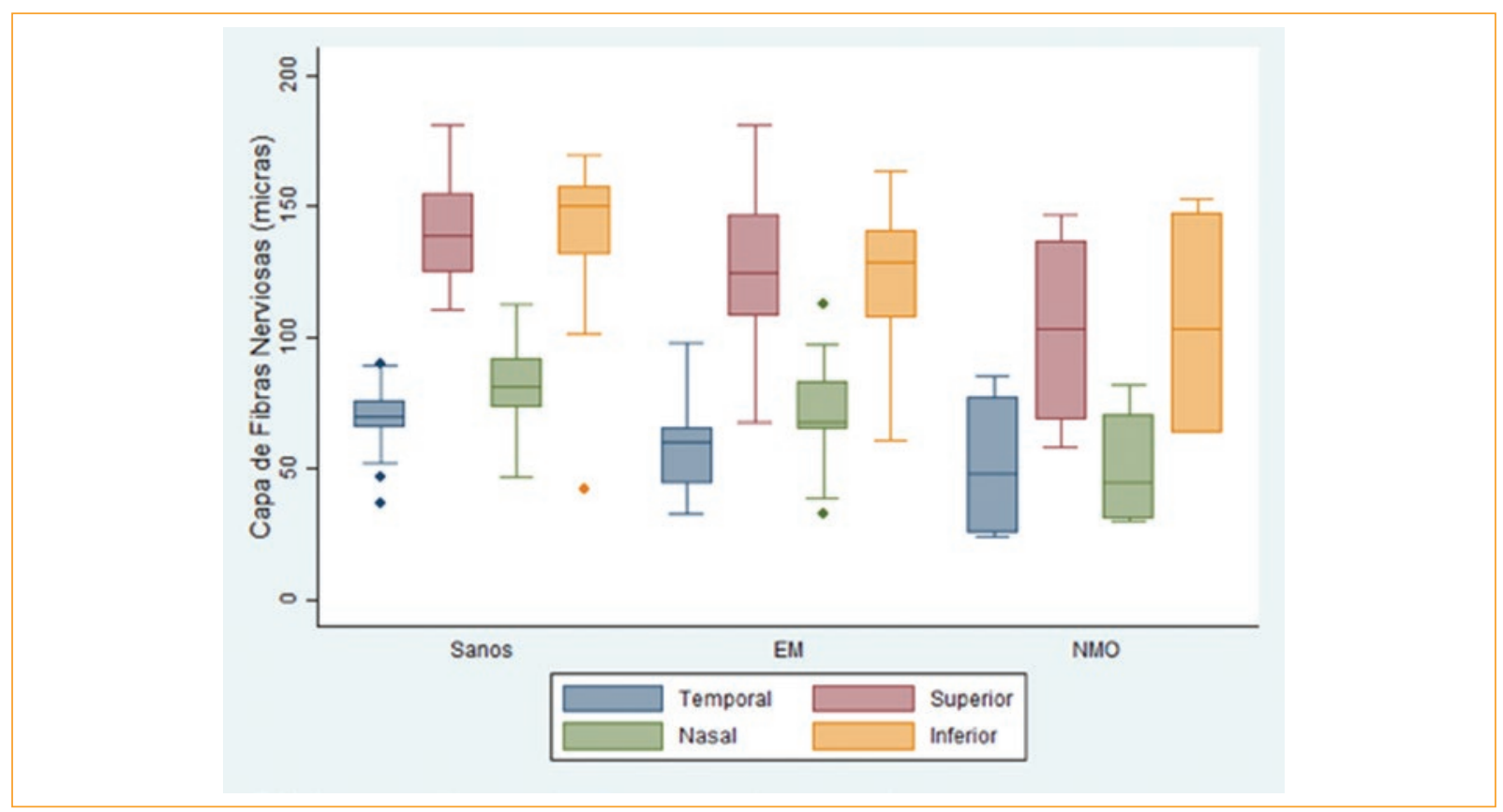

Figura 2. Grosor en $\mu \mathrm{m}$ de la capa de fibras nerviosas por cuadrantes por diagnóstico ( $0=$ sanos, $1=\mathrm{EM}, 2=\mathrm{NM} 0$ ). EM: esclerosis múltiple; NMO: neuromielitis óptica.

Basado en estudios previos se reporta que el espesor medio de la CFN en EM es de $92 \mu \mathrm{m}$, y en ojos de pacientes sanos es de $105 \mu \mathrm{m}^{17}$. Nuestros resultados reportan un grosor de $94 \mu \mathrm{m}$ en pacientes con EM y $110 \mu \mathrm{m}$ en pacientes sanos, lo cual concuerda con los estudios previamente mencionados. Este valor es 


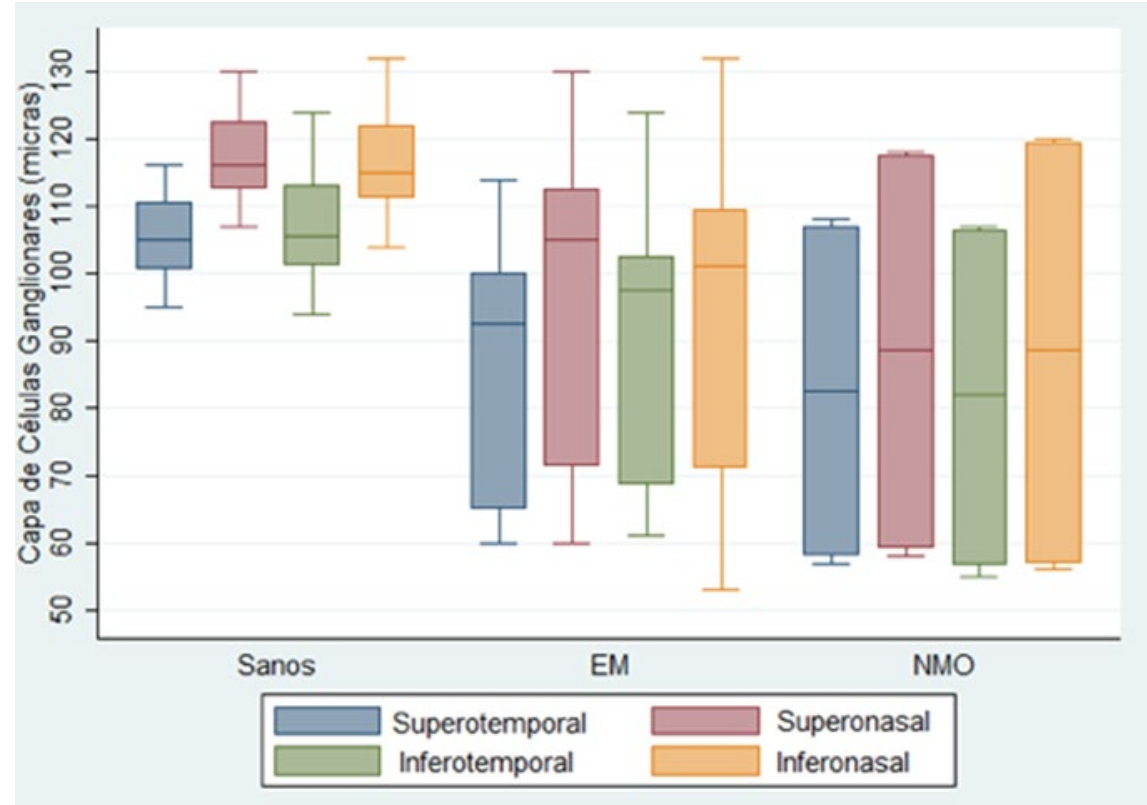

Figura 3. Grosor en $\mu \mathrm{m}$ de la capa de células ganglionares por cuadrante.

EM: esclerosis múltiple; NMO: neuromielitis óptica.

mucho menor en pacientes con NMO $(76.5 \mu \mathrm{m})$, tanto en este estudio como para el valor previamente reportado ${ }^{2,13}$.

Se ha descrito la posible utilidad de la OCT en el diagnóstico de NMO. Esta depende de que el espesor de la capa de células ganglionares superotemporales sea menor a $62 \mu m^{2,16}$; sin embargo, este límite o punto de corte no fue identificado en nuestra población, que fue de 82.5 (RIQ: 49) $\mu \mathrm{m}$.

Ya que la mácula consta principalmente de cuerpos de células ganglionares, la evaluación del volumen macular permite determinar si la pérdida axonal (medida por el espesor de la CFN) se asocia con la degeneración neuronal18-20. En este estudio se determinó mayor pérdida de células ganglionares a nivel macular en los cuadrantes superotemporal e inferonasal en los pacientes con EM, y superonasal e inferonasal en los pacientes con NMO, lo que se correlaciona con la pérdida de células ganglionares en los cuadrantes superior e inferior de la CFN a nivel del disco óptico, lo que explica la degeneración neuronal presente en estas dos enfermedades.

Es necesario aclarar que la poca evidencia disponible de la OCT con dominio espectral no es comparable con nuestros datos, dado que se ha descrito variabilidad en la medición, incluso entre diferentes marcas que usen la misma tecnología ${ }^{16,18}$.

La medición del espesor de la CFN y del grosor de células ganglionares merece un mayor esfuerzo de investigación, particularmente en estudios longitudinales, ya que en estos se ha descrito su utilidad pronóstica y evaluación terapéutica ${ }^{19,21}$.

\section{Conclusiones}

Los pacientes con NMO y EM presentan pérdida en la CFN y en el complejo de células ganglionares. Los pacientes con NMO presentan mayor pérdida en la CFN que los pacientes con EM. El patrón de pérdida (en relación a los cuadrantes) tiende a ser más generalizado en la NMO (afectando los cuadrantes inferior-superior) y tiende a afectar principalmente los cuadrantes temporales en la EM. En pacientes con NMO, la diferencia en la CFN entre ambos ojos (el que tuvo neuritis y el que no ha tenido) podría ayudar a diferenciar entre la NMO y la EM. En la EM se puede documentar pérdida en la CFN, incluso en ojos que no tienen antecedentes de neuritis óptica clínica.

Puesto que es un estudio de serie de casos, no puede establecerse asociación o predicción en términos de progresión o gravedad. 
El comportamiento del compromiso de las dos capas medidas por cuadrantes es similar al reportado por otros autores, donde se puede establecer sospecha de compromiso por NMO si el grosor de la capa de células ganglionares en el cuadrante superotemporal es menor a $62 \mu \mathrm{m}$. Sin embargo, el grosor encontrado en nuestros pacientes, aunque se encuentra disminuido comparado con sujetos sanos, no alcanzó dicho umbral, lo cual puede ser reflejo del tamaño de la muestra y del tipo de diseño de nuestro estudio, que hacen difícil evaluar el espectro de la enfermedad.

La baja prevalencia de pacientes con una enfermedad desmielinizante como la EM y la NMO limitó el tamaño de la muestra de este estudio. Cabe resaltar que este estudio solo se realizó en pacientes sin neuritis óptica activa, lo que no permite conocer el papel de la OCT SD en los momentos agudos de estas dos enfermedades.

Ante toda información disponible de medidas de la CFN y de la capa de células ganglionares con técnica $\mathrm{SD}$, nuestros resultados aportan información valiosa en relación con el comportamiento de la EM y la NMO y su compromiso ocular.

\section{Conflicto de intereses}

Los autores declaran no tener conflictos de interés en el presente trabajo de investigación ni haber recibido financiación para el mismo.

\section{Responsabilidades éticas}

Protección de personas y animales. Los autores declaran que los procedimientos seguidos se conformaron a las normas éticas del comité de experimentación humana responsable y de acuerdo con la Asociación Médica Mundial y la Declaración de Helsinki.

Confidencialidad de los datos. Los autores declaran que han seguido los protocolos de su centro de trabajo sobre la publicación de datos de pacientes.

Derecho a la privacidad y consentimiento informado. Los autores han obtenido el consentimiento informado de los pacientes y/o sujetos referidos en el artículo. Este documento obra en poder del autor de correspondencia.

\section{Bibliografía}

1. Kidd P. Multiple Sclerosis, An Autoimmune Inflammatory Disease: Prospects for its Integratiev Management. Altern Med Rev. 2001;6(6):26.

2. Bennett JL, de Seze J, Lana-Peixoto M, Palace J, Waldman A, Schippling S, et al. Neuromyelitis optica and multiple sclerosis: Seeing differences through optical coherence tomography. Mult Scler. 2015;21(6): 678-88.

3. Moriarty JA. Optic neuritis. Minn Med. 1968;51:1259-63.

4. Balk LJ, Coric D, Bijvank JAN, Killestein J, Uitdehaag BMJ, Petzold A. Retinal atrophy in relation to visual functioning and vision-related quality of life in patients with multiple sclerosis. Mult Scler. 2018; 24(6):767-76.

5. Kallenbach K, Frederiksen J. Optical coherence tomography in optic neuritis and multiple sclerosis: A review. Eur J Neurol. 2007;14:841-9.

6. Reichel E, Ho J, Duker JS. OCT Units: Which One Is Right for Me? Rev Ophthalmol. 2009;16(9):62.

7. Oct A, System SLO. Advanced OCT/SLO System.

8. Elong Ngono A, Lepetit M, Reindl M, Garcia A, Guillot F, Genty A, et al Decreased Frequency of Circulating Myelin Oligodendrocyte Glycoprotein B Lymphocytes in Patients with Relapsing-Remitting Multiple Sclerosis. J Immunol Res. 2015;2015:673503.

9. Toosy AT, Mason DF, Miller DH. Optic neuritis. Lancet Neurol. 2014;13(1): 83-99.

10. Chiquete E, Navarro-bonnet J, Ayala-armas R, Gutiérrez-gutiérrez N, Solórzano-meléndez A, Rodríguez-tapia $D$, et al. Neuromielitis óptica: actualización clínica. Rev Neurol. 2010;51(5):289-94

11. Lucchinetti CF, Mandler RN, Mcgavern D, Bruck W, Gleich G, Ransohoff RM, et al. A role for humoral mechanisms in the pathogenesis of Devic's neuromyelitis optica. Brain. 2002;1450-61.

12. Charcot J-M. Histologie de la sclérose en plaques, leçon faite à l'hospice de la Salpêtrière par M. Charcot et recueillie par M. Bourneville. 1887

13. Klawiter EC. Optical coherence tomography differs in neuromyelitis optica compared with multiple sclerosis. Neurology. 2009;72:(12): 1077-82.

14. Peng $\mathrm{C}$, Wang $\mathrm{W}, \mathrm{Xu} \mathrm{Q}$, Yang $\mathrm{M}$, Zhou H, Zhao S. Thickness of macular inner retinal layers and peripapillary retinal nerve fibre layer in neuromyelitis optica spectrum optic neuritis and isolated optic neuritis with one episode. Acta Ophthalmol. 2017;95(6):583-90.

15. Outteryck O, Majed B, Defoort-dhellemmes S, Vermersch P. A comparative optical coherence tomography study in neuromyelitis optica spectrum disorder and multiple sclerosis. Mult Scler. 2015;21(14): 1781-93.

16. Park K, Kim J, Oh SY. Analysis of spectral domain optical coherence tomography measurements in optic neuritis: differences in neuromyelitis optica, multiple sclerosis, isolated optic neuritis and normal healthy controls. Acta Ophthalmol. 2014;92(1):e57-65.

17. Balk LJ, Petzold A. Current and future potential of retinal optical coherence tomography in multiple sclerosis with and without optic neuritis. Neurodegener Dis Manag. 2014;4(2014):165-76.

18. Lamirel C, Newman NJ, Biousse V. Optical coherence tofmography (OCT) in optic neuritis and multiple sclerosis. Rev Neurol (Paris) [Internet]. Elsevier Masson SAS; 2010;166(12):978-86.

19. Balk LJ, Cruz-Herranz A, Albrecht P, Arnow S, Gelfand JM, Tewarie P, et al. Timing of retinal neuronal and axonal loss in MS: a longitudinal OCT study. 2016; 263(7):1323-31.

20. Petzold A. Optical Coherence Tomography to Assess Neurodegeneration in Multiple Sclerosis. Methods Mol Biol. 2016;1304:131-41.

21. Brandt AU, Martinez-lapiscina EH, Nolan R, Saidha S. Monitoring the Course of MS With Optical Coherence Tomography. Curr Treat Options Neurol. 2017;19(4):15. 